\title{
A systematic review and meta-analysis of incision and seton drainage in the treatment of high perianal abscess
}

\author{
Yuying Shi, Congcong Zhi, Yicheng Cheng, Lihua Zheng \\ Department of Anorectal Diseases, China-Japan Friendship Hospital, Beijing, China \\ Contributions: (I) Conception and design: Y Shi; (II) Administrative support: C Zhi; (III) Provision of study materials or patients: Y Cheng; (IV) \\ Collection and assembly of data: Y Shi, C Zhi, Y Cheng; (V) Data analysis and interpretation: Y Shi, L Zheng; (VI) Manuscript writing: All authors; \\ (VII) Final approval of manuscript: All authors. \\ Correspondence to: Lihua Zheng. Department of Anorectal Diseases, China-Japan Friendship Hospital, Beijing 100029, China. \\ Email: btv1262021@163.com.
}

Background Surgery is the clinically preferred treatment for high perianal abscesses. Incision and seton drainage improve the cure rate and reduce recurrence. We aimed to systematically evaluate the clinical effect and safety of incision and seton drainage in the treatment of high perianal abscess.

Methods: China Knowledge Network (CNKI), WanFang database, VIP database, PubMed, and Cochrane Library were searched and all relevant Chinese and English language documents until July 2021were retrieved. All records that described randomized clinical trials (RCTs) of incision and seton drainage for the treatment of high perianal abscess were eligible. Documents that met the inclusion criteria were evaluated for bias using the Cochrane Collaboration Risk Evaluation Standard, and Revman5.4 software was used to analyze the data.

Results: Fourteen RCTs were included. The results of nine studies showed that the clinical cure rate of the incision-seton group was higher than that of the incision-drainage group $(\mathrm{P}<0.05)$. Seven studies showed that the wound healing time of the incision-seton group was shorter than that of the incision-drainage group $(\mathrm{P}<0.05)$. Four studies showed that the visual analogue scale (VAS) score of the incision-seton group was lower than that of the incision-drainage group $(\mathrm{P}<0.05)$. Five studies showed that the Wexner score of the incision-seton group was lower than that of the incision-drainage group $(\mathrm{P}<0.05)$. Six studies showed that the formation rate of anal fistula in the incision-seton group was lower than that in the incision-drainage group $(\mathrm{P}<0.05)$. Six studies demonstrated that the recurrence rate of abscess in the incision-seton group was lower than that in the incision-drainage group $(\mathrm{P}<0.05)$. Seven studies showed that the incidence of adverse events in the incision-seton group was lower than that in the incision-drainage group $(\mathrm{P}<0.05)$. Five studies demonstrated that the length of stay in the incision-seton group was shorter than that of the incisiondrainage group $(\mathrm{P}<0.05)$.

Discussion: The choice of surgical methods in clinical research has always been controversial. The incision-seton method can effectively and safely treat high perianal abscess. However, the results of this meta-analysis still leave some gaps in the evidence. More large-sample, high-quality, and multi-center RCTs are needed.

Keywords: Incision-seton therapy; incision-drainage therapy; high perianal abscess; meta-analysis

Submitted Jul 19, 2021. Accepted for publication Sep 16, 2021.

doi: 10.21037/apm-21-2229

View this article at: http://dx.doi.org/10.21037/apm-21-2229 


\section{Introduction}

High perianal abscess refers to a perianal abscess with the levator ani muscle as the boundary, where the infection space of the abscess involves the deep retrorectal space and/ or the submucosal space of the rectum and/or the perianal abscess above the levator anus of the pelvic rectal space abscess (1). The 2016 edition of the American College of Colorectal Surgeons' "Guidelines for the Treatment of Perianal Abscess, Anal Fistula, and Rectovaginal Fistula" points out that incision-drainage is the most important method for the treatment of perianal abscess (2). However, it is difficult to apply simple incision and drainage for high perianal abscesses in clinical practice (3). On the one hand, in terms of diagnosis, because high perianal abscesses often invade the levator ani muscle, and their location is deeper in the intestinal cavity, the distance from the anal edge is higher, and there are often no obvious signs outside the perianal area. Perianal abscesses are easy to be misdiagnosed, treatment is often delayed, which can lead to spreading of pus and even severe complications such as sepsis and necrotizing fasciitis (4). On the other hand, in terms of treatment, although incision and drainage can further drain the pus and avoid subsequent extension of the infection, because the perianal abscess involves a higher position of the anal muscle layer, it may be difficult to use a low incision and drainage to achieve thorough drainage and avoid the spread of inflammation and infection (5). As such, due to the incomplete treatment of necrotic abscess cavity and infective space, perianal swelling and pain are often recurring subsequently or, if left behind, form high complex anal fistulas $(6,7)$.

Surgery is the first choice for the clinical treatment of high perianal abscess. The choice of surgical methods has always been controversial in clinical research (8). The main purpose of treatment is to cure perianal abscesses, while at the same time minimizing the patient's pain, protect anal sphincter function and reduce the recurrence of high anal fistulas $(6,9)$. At present, the most common surgical procedure in clinical practice is low incision and high seton-hanging (6). Seton-drainage refers to utility of seton through the fistula channels to drainage pus. Compared with common drainage, seton used in drainage is very thin which has little influence on the healing of fistula channel.

The clinical application of incision-seton in the treatment of high abscesses has advantages such as improving the cure rate and reducing recurrence. However, due to the small sample size of a single study and the inconsistent results between studies, this article aims to analyze the relevant clinical publications by systematic analysis of clinical data to provide a comprehensive, systematic and standardized comparison of incision-seton with incision-drainage, and systematic evaluation of the clinical effect and safety of incision-seton in the treatment of high perianal abscesses.

We present the following article in accordance with the PRISMA reporting checklist (available at https://dx.doi. org/10.21037/apm-21-2229).

\section{Methods}

\section{Database}

Chinese and foreign databases were searched. The Chinese databases were: China Knowledge Network (CNKI), WanFang database, VIP database, and the foreign language databases were: Cochrane Library and PubMed.

\section{Retrieval strategy}

Search terms for retrieval using the Chinese databases were: "perianal abscess" or "anal carbuncle" or "perianorectal abscess" or "perianorectal abscess" and "high position", "seton" and "incision and drainage" and "pelvic rectal fossa abscess" or "retrorectal space abscess" or "high perianal abscess", "thread hanging", and the foreign language search formula was "Perianal Abscess and Seton". From the establishment of each database to July 10, 2021, a comprehensive search of the Chinese and English databases was undertaken. The treatment group involved treatment of high perianal abscesses with low incision and high suture (including high solid suture, virtual suture, virtual and solid suture suture). The control group involved incision and drainage treatment.

\section{Inclusion criteria}

\section{Study types}

Prospective, randomized, controlled trials (RCTs). According to Cochrane criteria, trials were included regardless of whether or not blinding was used not, and only records in Chinese and English were included.

\section{Research subjects}

Patients who were diagnosed with high perianal abscess and underwent surgical treatment. Diagnostic criteria: Based on the diagnostic criteria of anal carbuncle [Treatment 
of Common Diseases (TCD) code: BWG040] in the "Guidelines for the Diagnosis and Treatment of Common Diseases in the Department of Anorectal Diseases of the Chinese Society of Traditional Chinese Medicine", high perianal abscesses are bounded by the levator anus muscle, and the infection space involves the deep posterior space and/or the submucosal space of the rectum and/or the perianal abscess above the levator ani muscle of the pelvicrectal space abscess (10).

\section{Intervention measures}

The patients in treatment group received incisionseton surgery (including real seton, virtual seton, and a combination of loose and cutting seton).

The patients in the control group received incisiondrainage surgery. According to the 2016 edition of the American College of Colorectal Surgeons' "Guidelines for the Treatment of Perianal Abscess, Anal Fistula, and Rectovaginal Fistula", incision and drainage is the most important method for the treatment of perianal abscess (2).

\section{Outcome measures}

(I) Cure rate, (II) wound healing time, (III) visual analogue scale (VAS) pain score, (IV) anal function Wexner score, (V) anal fistula formation rate, (VI) abscess recurrence rate, (VII) adverse event rate, and (VIII) hospitalization time.

\section{Exclusion criteria}

(I) Insufficient data, duplicate publication, non-RCT studies such as reviews, expert opinions, description of mechanisms, and case reports; (II) animal, pharmacological or pharmacokinetic experiments; (III) the treatment group received other treatment methods at the same time; (IV) included in the study were patients with cardiocerebrovascular or other serious organic diseases, mental illness, or complications.

\section{Literature selection strategy}

Two reviewers screened the literature and performed the quality evaluations independently and then cross-checked them. If there were any differences, they were resolved through discussion, and if necessary, a third party was consulted. The inclusion criteria were evaluated according to the Cochrane Collaboration Risk Evaluation Standards, including whether studies were randomized, whether allocation concealment was achieved, whether blinding was used, whether the outcome data were complete, whether the results of the study were selectively reported and any other sources of bias.

\section{Statistical analysis}

Revman5.4 software was used for data analysis. First, a heterogeneity test was performed by Revman 5.4 software. If $\left(\mathrm{P}>0.01, \mathrm{I}^{2}<50 \%\right)$ this indicated that there were no statistically heterogeneous differences between the studies, and that use of a fixed model effects for meta-analysis were appropriate. If $\mathrm{P}<0.01$ and $\mathrm{I}^{2}>50 \%$, this indicated that there was significant heterogeneity, and the reasons for the heterogeneity were searched for and eliminated as much as possible. If the reasons for the heterogeneity could not be found, a random effects model meta-analysis was used. Risk of literature bias was evaluated using a funnel chart.

\section{Results}

\section{Literature search results}

A total of 104 documents in English were found; after reading the title and abstract, all documents were included. A total of 130 Chinese language articles were retrieved from the VIP database, CNKI database, and WanFang database. Duplicates were removed, leaving a total of 69 articles. Forty-eight articles did not meet eligibility criteria, leaving 21 articles. There are 14 RCTs and 7 retrospective studies, and these retrospective studies were excluded. A total of 14 articles reporting prospective RCTs were included in this meta-analysis. The literature selection process and results are shown in Figure 1.

\section{Basic characteristics of the included studies}

A total of 14 studies were included in this article. Because the articles did not fully cover clinical efficacy (including statistics of indicators such as cure rate and recurrence rate), postoperative healing time, and anal function score indicators, we used separate statistical analyses. Among the 14 studies, nine studies with a total of 721 patients statistically compared surgical cure rates; seven studies with a total of 563 patients statistically compared wound healing time; four studies with a total of 266 patients statistically compared VAS pain scores; five studies with a total of 332 patients statistically compared Wexner anal function scores; six studies with a total of 424 patients statistically compared 


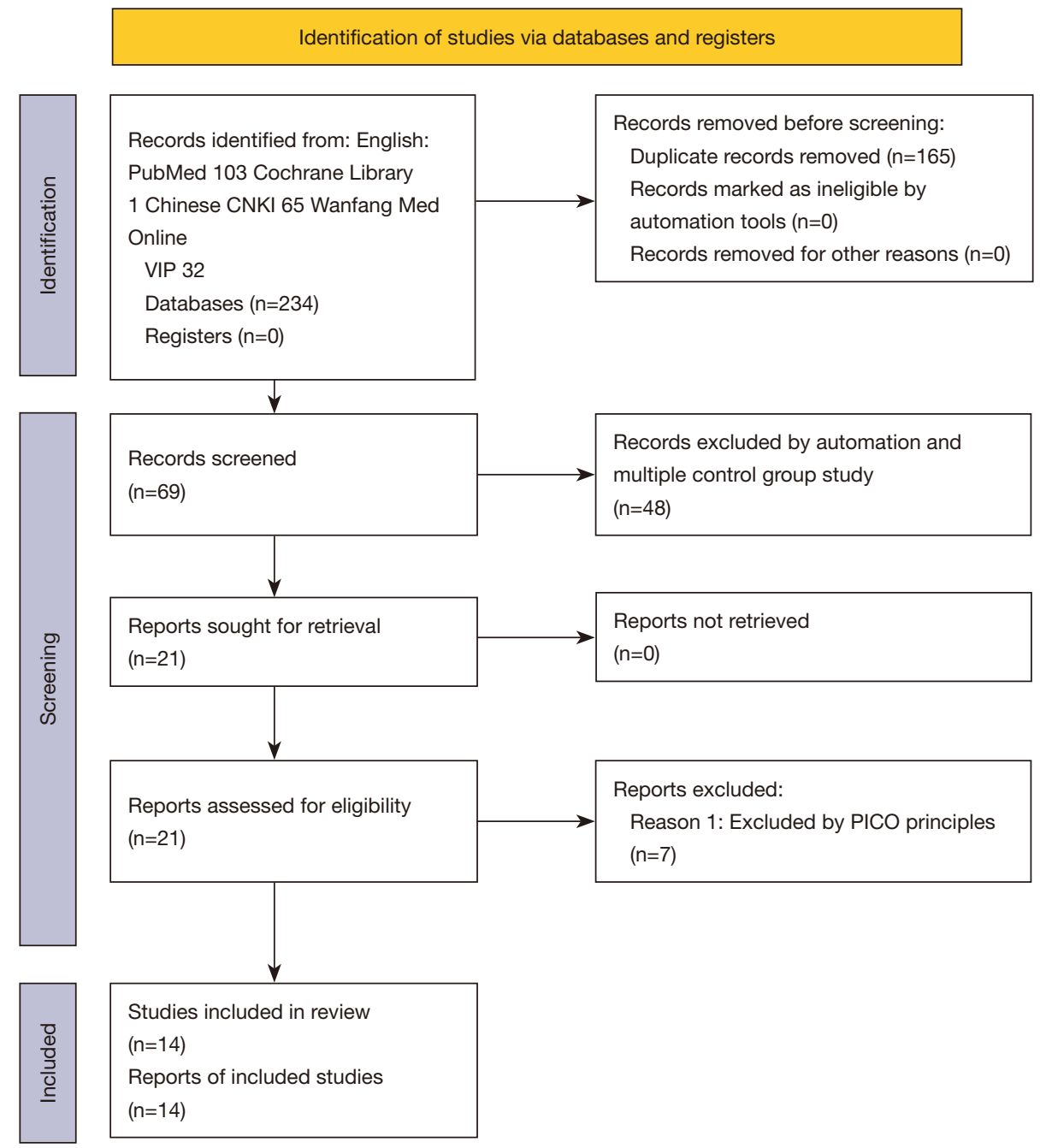

Figure 1 Flowchart of study selection. PICOS, participants, intervention, control, outcome and study design.

anal fistula formation rates; six studies with a total of 406 patients statistically compared abscess recurrence rates; seven studies with a total of 463 patients statistically compared the incidence of adverse events; five studies with a total of 393 patients statistically compared length of stays. Basic characteristics of the included articles are shown in Table 1.

\section{Evaluation of the quality of studies}

All 14 studies were conducted in China. Of these, nine described the use of randomization methods and were rated as "low risk", and the other research groups did not report specific randomization methods and were rated as "undefined". None of the 14 studies clearly reported whether blinding was used. Five of them reported no cure rates and lacked statistics on clinical efficacy. None of the included studies showed selective bias (Figure 2).

\section{Results of meta-analysis}

\section{Cure rate}

A total of 9 studies with a total of 721 patients were included with cure rate as the outcome indicator $(12,14,16$ $19,21,23,24)$. The heterogeneity test results showed that the heterogeneity between the studies was large $(\mathrm{P}<0.0001$, $\left.\mathrm{I}^{2}=76 \%\right)$, and as such a random effects model was used for the combined analysis. The results showed that the cure rate of the incision-seton group was higher. The difference was statistically significant, relative risk $(\mathrm{RR})=2.76,95 \%$ 
Table 1 Basic characteristics of the literature included

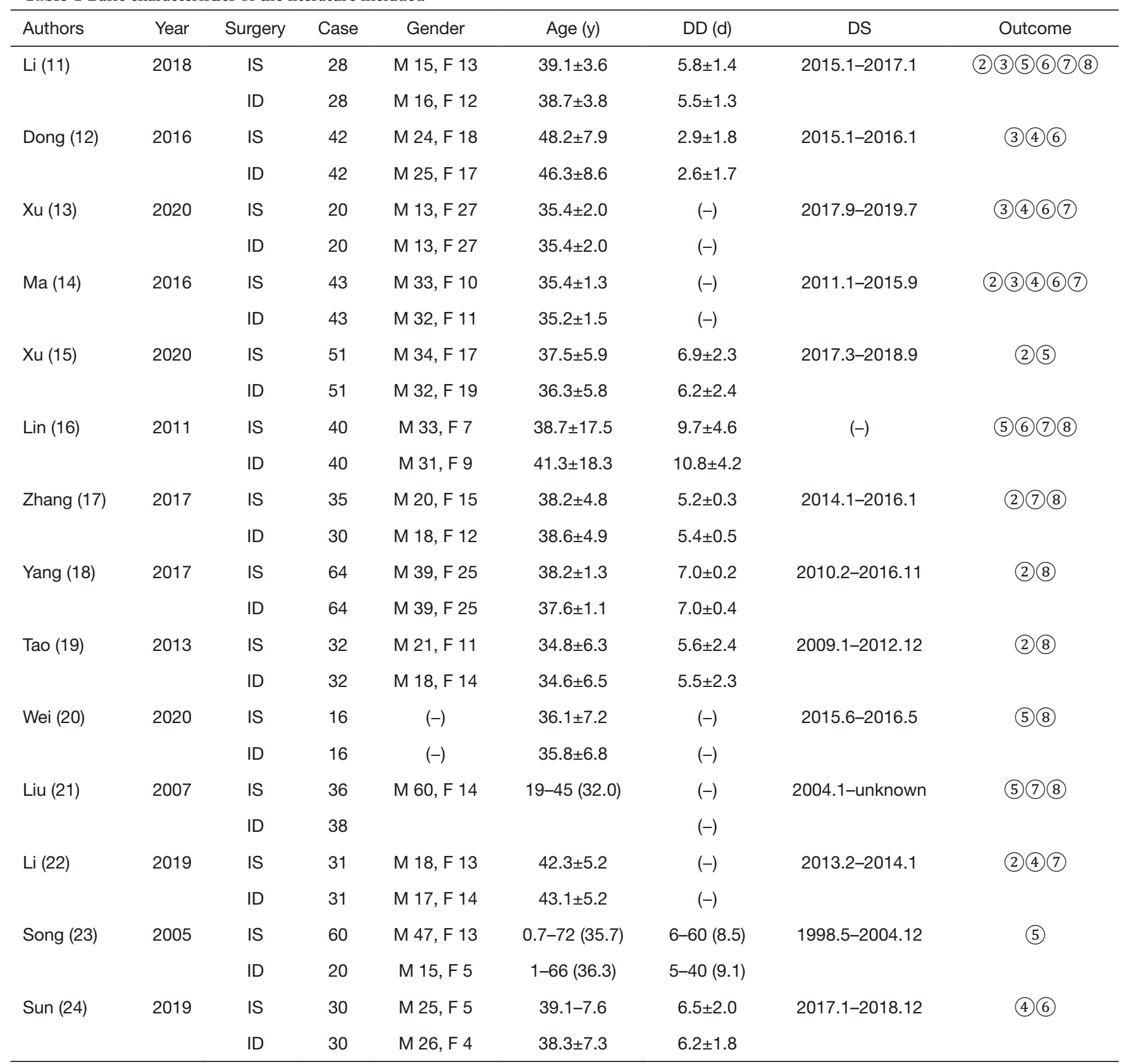

Notes: (1) healing rate; (2) wound healing time; (3) VAS pain score; (4) anal function Wexner score; (5) anal fistula formation rate; (6)abscess recurrence rate; (7) adverse event rate; (8) hospitalization time. IS, incision-seton; ID, incision-drainage; DD, duration of disease; DS, duration of study; M, male; F, female.

CI: (1.89, 4.04), $\mathrm{P}<0.00001$ (Figure 3).

\section{Wound healing time}

A total of seven studies with a total of 563 patients were included to compare wound healing time $(11,14,15,17$ -
19,22). The results of a heterogeneity test showed that the heterogeneity between the studies was large $(\mathrm{P}<0.00001$, $\left.I^{2}=97 \%\right)$, such that a random effects model was used for the combined analysis. The results showed that the wound healing time of the incision-seton group was longer than 


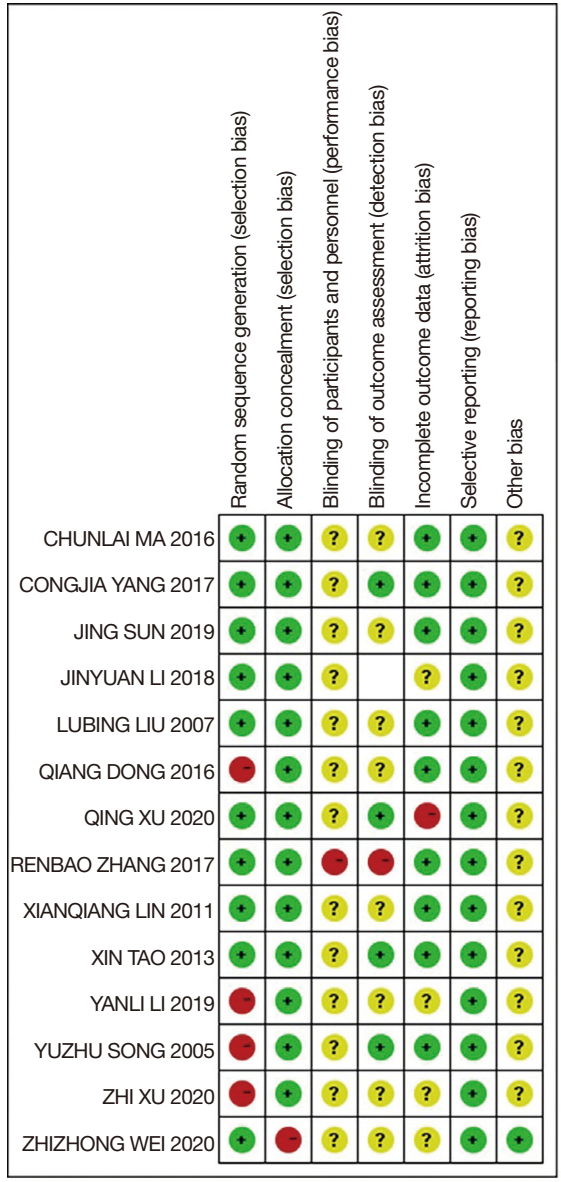

Figure 2 Risk of bias.

that of the incision-drainage group. The difference was statistically significant, mean difference $(\mathrm{MD})=-5.97,95 \%$ CI: (-7.88, -4.06), $\mathrm{P}<0.00001$ (Figure 4).

\section{VAS pain score}

A total of four studies were included to compare postoperative VAS pain scores, with a total of 266 patients (11-14). The heterogeneity test results showed that the heterogeneity between the studies was large $(\mathrm{P}<0.00001$, $\left.\mathrm{I}^{2}=99 \%\right)$. Therefore, a random effect model was used for the combined analysis. The results showed that the VAS score of the incision-seton group was higher than that of the incision-drainage group. The difference was statistically significant, $\mathrm{MD}=-1.30,95 \% \mathrm{CI}:(-2.16,-0.44), \mathrm{P}=0.003$ (Figure 5).

\section{Wexner anal function score}

A total of five studies with a total of 332 patients were included to compare the rate of postoperative anal fistula formation $(12-14,22,24)$. The results of a heterogeneity test showed that the heterogeneity among the studies was large $\left(\mathrm{P}<0.00001, \mathrm{I}^{2}=89 \%\right)$, and as such a random effects model was used for the combined analysis. The results showed that the Wexner score of the incision-seton group was higher than that of the incision-drainage group. The difference was statistically significant, $\mathrm{MD}=-0.82,95 \%$ CI: $(-1.05,-0.58)$, $\mathrm{P}<0.00001$ (Figure 6).

\section{Anal fistula formation rate}

A total of six studies with a total of 424 patients were included to compare the rate of postoperative anal fistula formation $(11,15,16,20,21,23)$. The heterogeneity test results showed that the heterogeneity between the studies was large $\left(\mathrm{P}=0.06, \mathrm{I}^{2}=53 \%\right)$, and as such a random effects model was used for the combined analysis. The results showed that the formation rate of anal fistula in the incision-seton group was higher than that in the incisiondrainage group. The difference was statistically significant, $\mathrm{RR}=0.14,95 \% \mathrm{CI}:(0.05,0.34), \mathrm{P}<0.0001$ (Figure 7).

\section{Abscess recurrence rate}

A total of six studies with a total of 406 patients were included to compare the recurrence rate of postoperative abscess (11-14,16,24). The results of a heterogeneity test showed that the homogeneity among the studies was better $\left(\mathrm{P}=0.98, \mathrm{I}^{2}=0 \%\right)$, and as such a fixed-effect model was used for the combined analysis. The results showed that the recurrence rate of abscess in the incision-seton group was higher than that of the incision-drainage group. The difference was statistically significant, $\mathrm{RR}=0.13,95 \% \mathrm{CI}$ : (0.06, 0.27), $\mathrm{P}<0.00001$ (Figure 8).

\section{Incidence of adverse events}

A total of seven studies with a total of 463 patients were included to compare the incidence of adverse events $(11,13,14,16,17,21,22)$. The heterogeneity test results showed that the homogeneity among the studies was relatively good $\left(\mathrm{P}=0.82, \mathrm{I}^{2}=0 \%\right)$. Therefore, a fixed-effect model was used for the combined analysis. The results showed that the incidence of adverse events in the incisionseton group was higher. The difference was statistically significant, $\mathrm{RR}=0.20,95 \% \mathrm{CI}:(0.09,0.43), \mathrm{P}<0.0001$ (Figure 9).

\section{Length of hospital stay}

A total of five studies with a total of 393 patients were 


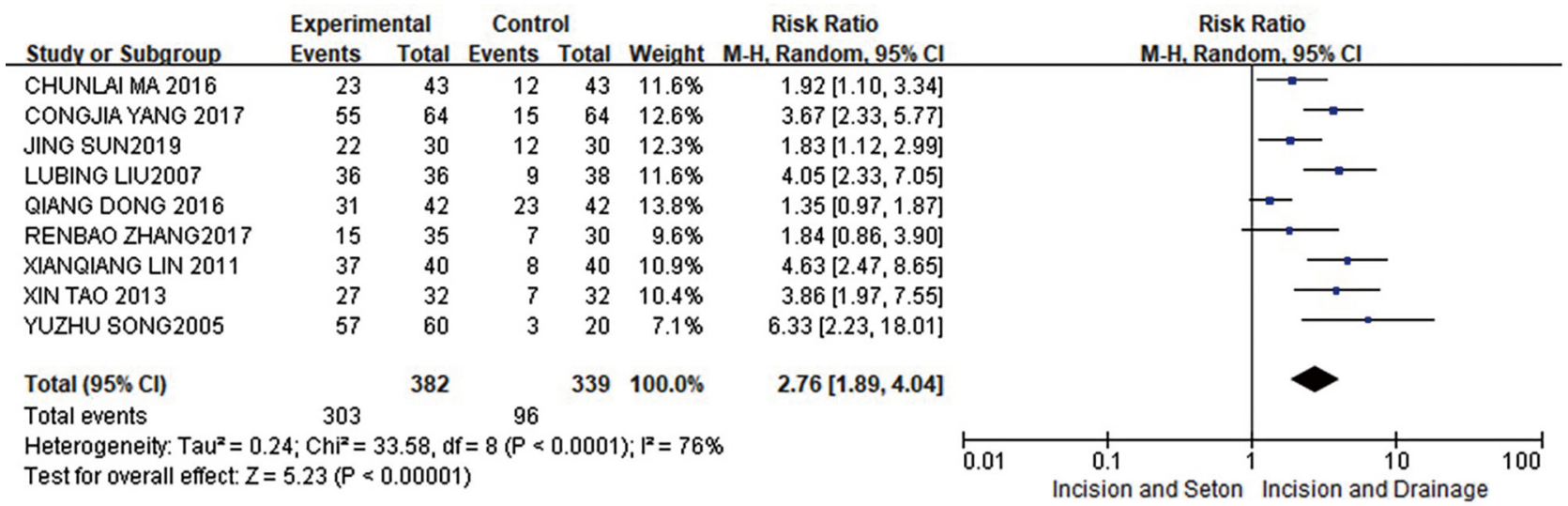

Figure 3 Analysis of cure rate.

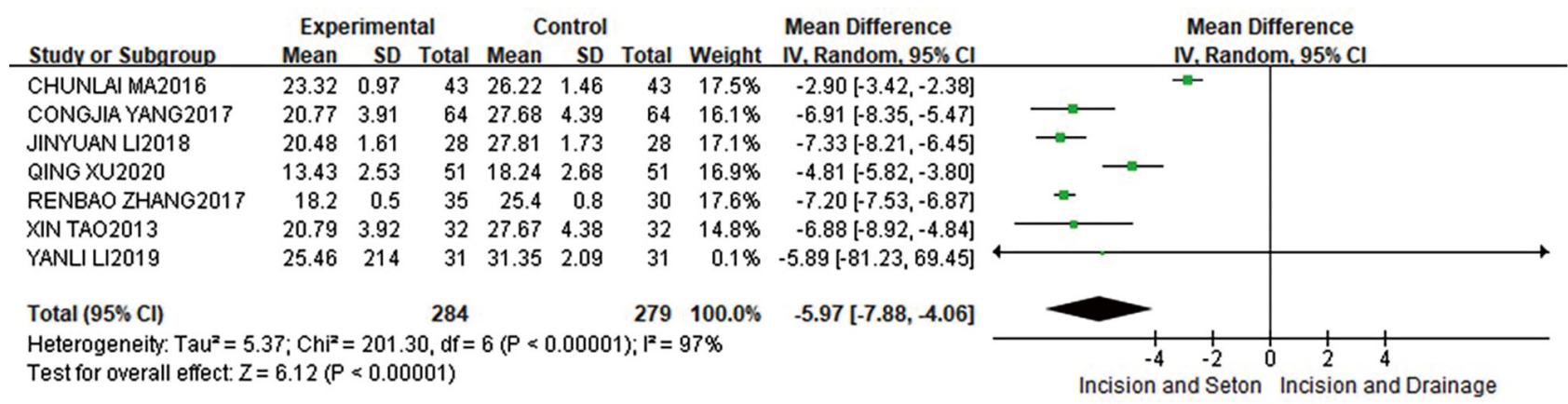

Figure 4 Analysis of wound healing time.

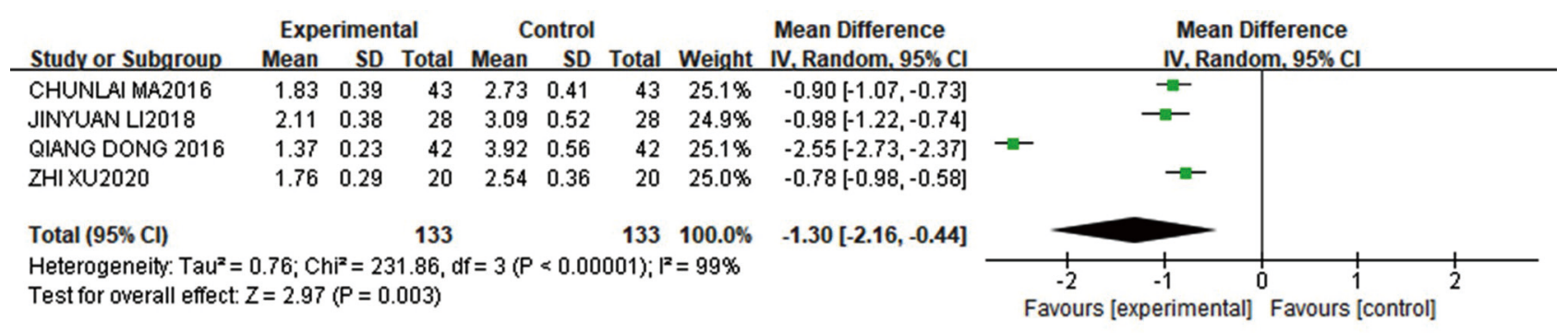

Figure 5 Analysis of VAS pain score. VAS, visual analogue scale.

included to compare the length of hospital stays (11,16-19). The heterogeneity test results showed that the heterogeneity between the studies was large $(\mathrm{P}<0.00001$, $\left.\mathrm{I}^{2}=96 \%\right)$, and as such a random effects model was used for the combined analysis. The results showed that the length of stay in the incision-seton group was longer than that of the incision-drainage group. The difference was statistically significant, $\mathrm{MD}=-8.36,95 \% \mathrm{CI}:(-10.87,-5.85), \mathrm{P}<0.00001$
(Figure 10).

\section{Publication bias analysis}

In this study, the effective rate of the incision-seton in the treatment of high perianal abscess and research bias were analyzed. The results indicated that some studies showed evidence of publication bias, which may be caused by the 


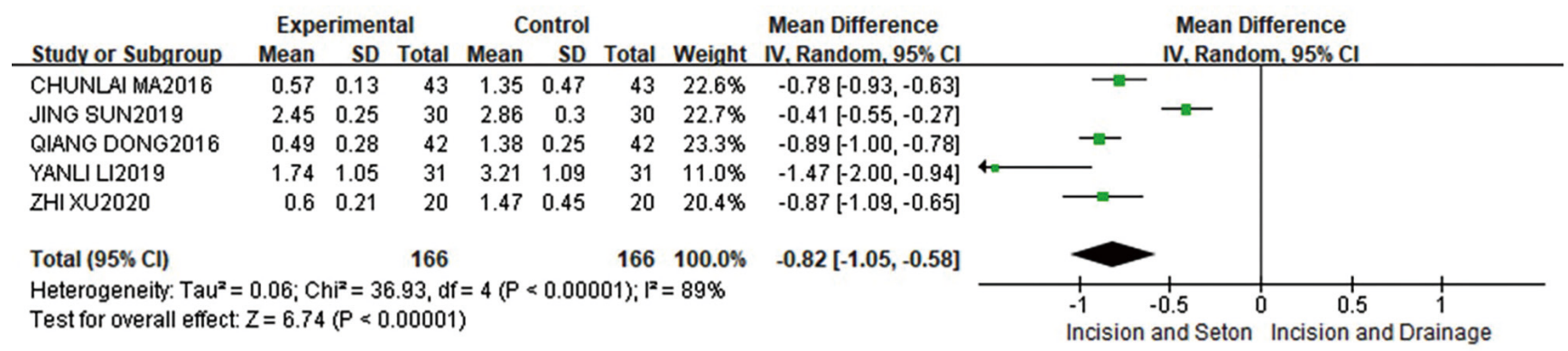

Figure 6 Analysis of Wexner anal function score.

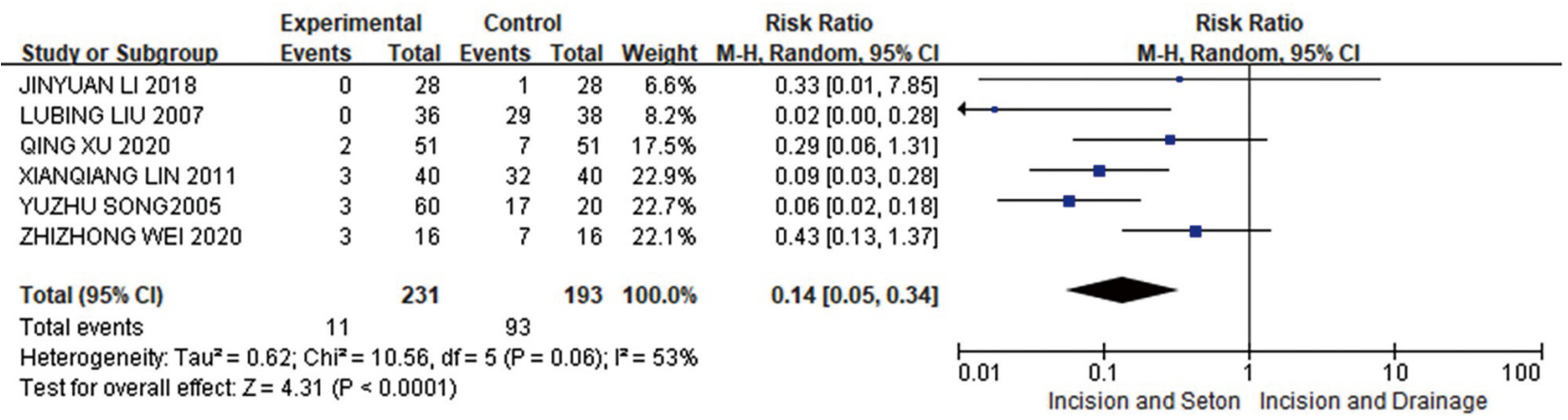

Figure 7 Analysis of anal fistula formation rate.

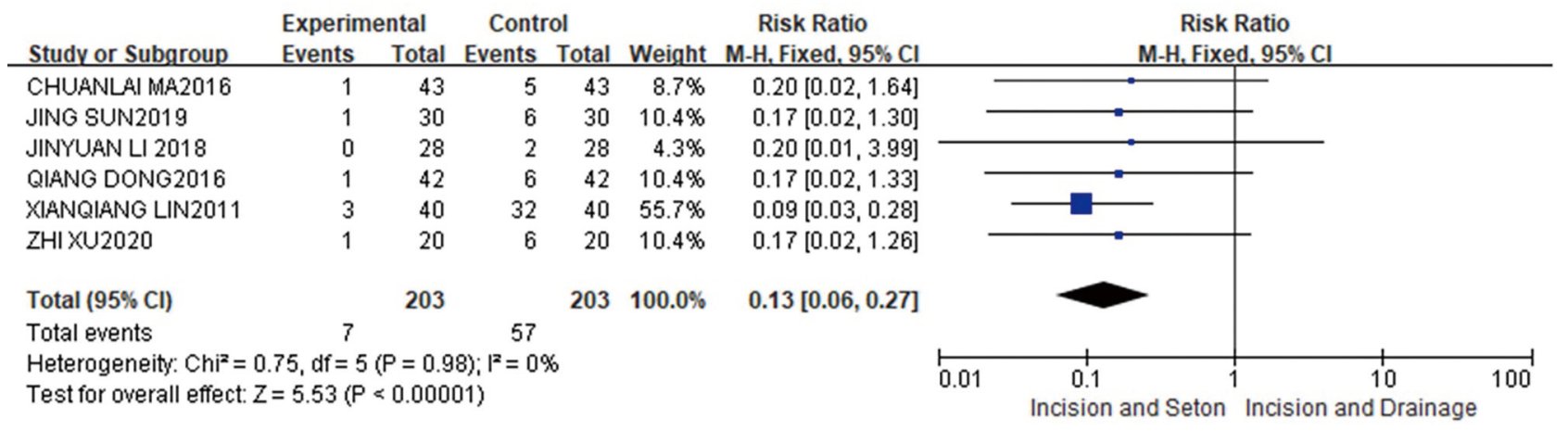

Figure 8 Analysis of abscess recurrence rate.

difficulty in publishing negative results (Figure 11).

\section{Discussion}

According to the diagnostic criteria of anal carbuncle (TCD code: BWG040) in the Chinese Society of Traditional Chinese Medicine's "Guidelines for the Diagnosis and Treatment of Common Diseases in the Area of Anorectal
Diseases relating to Traditional Chinese Medicine”, it is pointed out that high perianal abscess refers to the levator anus muscle as the boundary, and the infection space of the abscess involves the deep rectum. In this case the perianal abscess is located above the levator ani of the space and/or the submucosal space of the rectum and/or the abscess of the pelvic rectal space (15). Because high perianal abscesses often invade the levator ani muscle, their location is deeper 


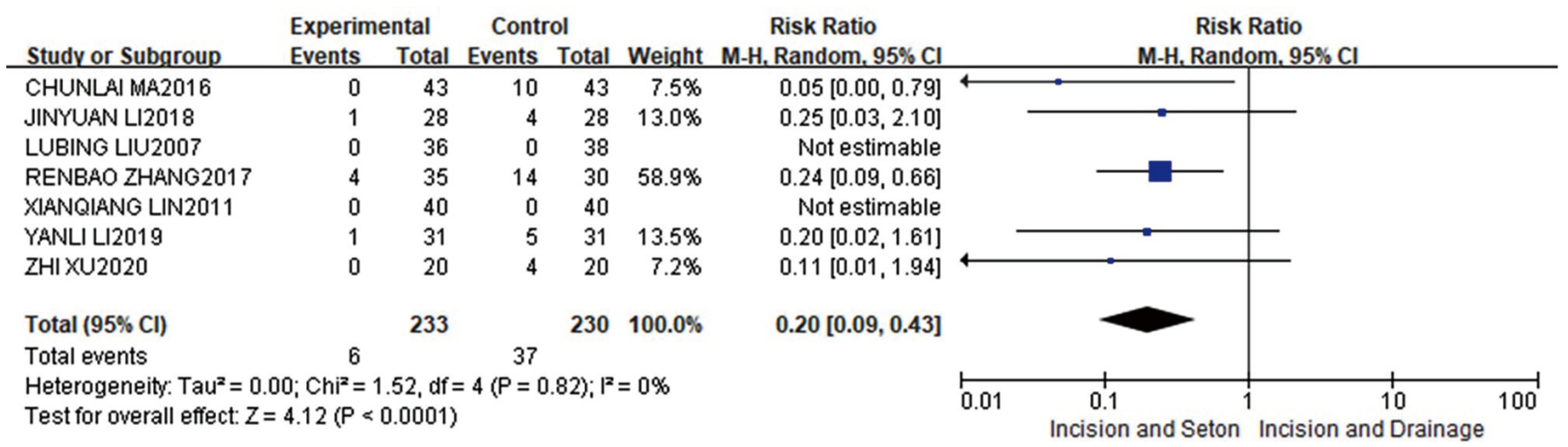

Figure 9 Analysis of Incidence of adverse events.

\begin{tabular}{lrrrrrrr} 
& \multicolumn{1}{c}{ Experimental } & \multicolumn{1}{c}{ Control } \\
Study or Subgroup & Mean & SD & Total & Mean & SD & Total & Weight \\
\hline CONGJIA YANG2017 & 13.41 & 3.11 & 64 & 19.35 & 3.68 & 64 & $20.6 \%$ \\
JINYUAN LI 2018 & 13.5 & 1.24 & 28 & 19.25 & 1.08 & 28 & $21.3 \%$ \\
RENBAO ZHANG2017 & 14.3 & 1.8 & 35 & 24.5 & 2.6 & 30 & $20.7 \%$ \\
XIANQIANG LIN 2011 & 28 & 5.5 & 40 & 43 & 6.3 & 40 & $17.6 \%$ \\
XIN TAO2013 & 13.45 & 3.12 & 32 & 19.34 & 3.65 & 32 & $19.7 \%$ \\
Total (95\% CI) & \multicolumn{7}{c}{199} \\
Heterogeneity: Tau $=7.59 ;$ Chi $^{2}=89.38$, df $=4(P<0.00001) ; I^{2}=96 \%$ \\
Test for overall effect: $Z=6.53(P<0.00001)$
\end{tabular}

Figure 10 Analysis of length of hospital stay.

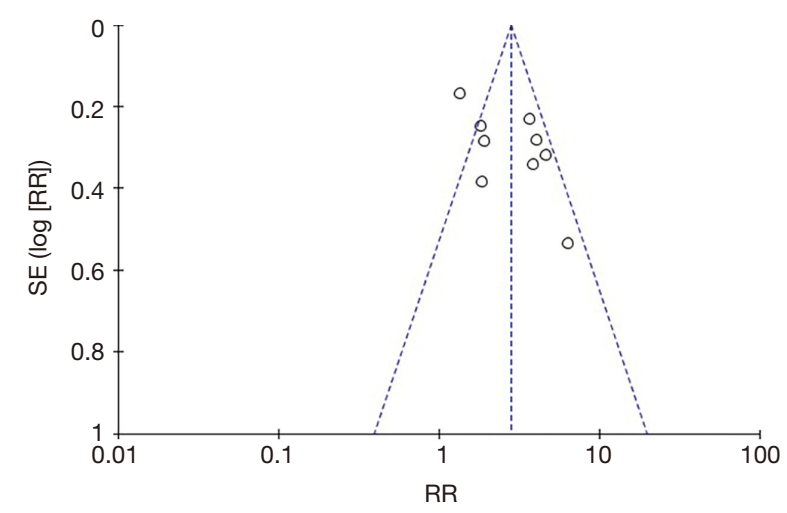

Figure 11 Analysis of publication bias.

in the intestinal cavity, and the distance from the anal edge is higher. There are often no signs outside the area of the perianal abscess, and as such it is easy for it to be misdiagnosed clinically or for a delay diagnosis to occur, leading to the spread of pus, if not serious complications such as sepsis and necrotizing fasciitis. Perioperative antibiotic therapy accelerates the healing of fistula, but the

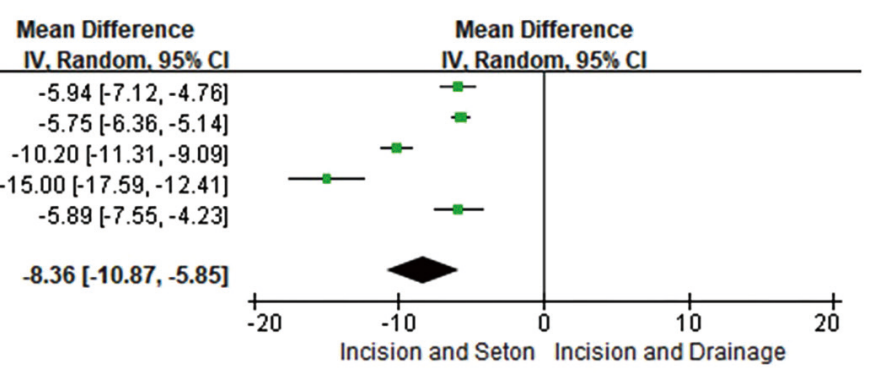

effect of antibiotic treatment alone is not ideal.

According to the 2016 edition of the American College of Colorectal Surgeons' "Guidelines for the Treatment of Perianal Abscess, Anal Fistula, and Rectovaginal Fistula”, incision and drainage is the most important method for the treatment of perianal abscess (16). However, in clinical practice it is difficult to apply simple incision and drainage for high perianal abscess. Although incision and drainage can further drain the pus and avoid further extension of the infection, because the perianal abscess involves a higher position of the anal ring muscle layer, complete drainage and avoidance of the spread of inflammation and infection may be difficult to achieve if the incision is low and. At the same time, due to incomplete treatment of the necrotic abscess in the cavity, perianal swelling and pain often recur subsequently or a high complex anal fistula develops.

Surgery is the first choice for clinical treatment of high perianal abscess. The choice of surgical methods in clinical research has always been controversial. The main purpose of treatment is to cure the perianal abscess, while at the same time minimizing the patient's pain, protecting the anal sphincter function and reducing the recurrence of high 
anal fistulas. This is especially important for high perianal abscess. At present, the most common clinical operation is low incision and high suture. This article systematically analyzes related published clinical data, comprehensively compares incision-seton and incision-drainage and systematically evaluates the clinical effect and safety of incision-seton in the treatment of high perianal abscess.

The surgical method of incision-seton is as follows: after administering anesthesia, a radial incision is performed at the most obvious location of the perianal abscess. After fully draining the pus, the index finger enters the abscess cavity deeply to separate the abscess cavity, the right-hand probe is directed to explore the abscess cavity, the left index finger penetrates the abscess cavity from the anus and guides the needle and fingers together to locate the inner opening of the abscess cavity. If the inner opening is protruded, the inner opening is cut once. If it is not protruded, the bottom of the abscess cavity should be explored. If the highest part of the cavity protrudes, the two sections are tightened using a rubber band. The surgical procedure corresponds to a process of chronic "strangulation" and drainage. Due to the continuous drainage of the rubber band, the purpose of smooth drainage is achieved. The continuous tightening stimulation of the rubber band applies to the sphincter and surrounding area. Tissue adhesion, to achieve the purpose of cutting and drainage, adhesion. Eventually the cure of perianal abscess is achieved and the risk of postoperative anal incontinence is reduced.

A total of 14 RCTs meeting the eligibility criteria were included in this study. Some articles did not fully cover clinical efficacy (including statistics of indicators such as cure rate and recurrence rate), postoperative healing time, and anal function scores. Therefore, the authors used separate statistical analyses for each indicator. The results show that the incision-seton group had obvious advantages over the incision-drainage group. The surgical cure rate for the treatment of high perianal abscess was high, the wound healing time was short, pain was short-lived, hospital stays were short, and there were few postoperative adverse reactions. Functional scores, postoperative recurrence rates, and anal fistula formation rates were low, which provides credible medical evidence for the use of incision-seton in high perianal abscesses. With these results, we recommend that for patients with high perianal abscesses, incision and seton drainage should be first line choice.

However, the results of this meta-analysis still leave some gaps in the evidence. When the quality of the studies reported in the articles was evaluated using the Cochrane quality assessment tool, it became apparent that none of the studies reported on whether blinding was used in this study.

Lack of blinding suggests a potential for bias, which may have affected the results and the strength of the evidence. Therefore, for the evaluation of the effectiveness of incision and thread-drawing in the treatment of high perianal abscess, more large-sample, high-quality, and multi-center RCTs are needed. The authors recommend that clinical investigators use blinding and allocation concealment in future trials to comprehensively enhance the accuracy and credibility of the research results.

\section{Acknowledgments}

Funding: None.

\section{Footnote}

Reporting Checklist: The authors have completed the PRISMA reporting checklist. Available at https://dx.doi. org/10.21037/apm-21-2229

Conflicts of Interest: All authors have completed the ICMJE uniform disclosure form (available at https://dx.doi. org/10.21037/apm-21-2229). The authors have no conflicts of interest to declare.

Ethical Statement: The authors are accountable for all aspects of the work in ensuring that questions related to the accuracy or integrity of any part of the work are appropriately investigated and resolved.

Open Access Statement: This is an Open Access article distributed in accordance with the Creative Commons Attribution-NonCommercial-NoDerivs 4.0 International License (CC BY-NC-ND 4.0), which permits the noncommercial replication and distribution of the article with the strict proviso that no changes or edits are made and the original work is properly cited (including links to both the formal publication through the relevant DOI and the license). See: https://creativecommons.org/licenses/by-nc-nd/4.0/.

\section{References}

1. Zhou Y, Gao H, Hua H, et al. Clinical effectiveness of matrine sitz bath in treating perianal infection after chemotherapy for acute leukemia. Ann Palliat Med 2020;9:1109-16. 
2. Vogel JD, Johnson EK, Morris AM, et al. Clinical Practice Guideline for the Management of Anorectal Abscess, Fistula-in-Ano, and Rectovaginal Fistula. Dis Colon Rectum 2016;59:1117-33.

3. Malik AI, Nelson RL, Tou S. Incision and drainage of perianal abscess with or without treatment of anal fistula. Cochrane Database Syst Rev 2010:CD006827.

4. Sahnan K, Adegbola SO, Tozer PJ, et al. Perianal abscess. BMJ 2017;356:j475.

5. Cox SW, Senagore AJ, Luchtefeld MA, et al. Outcome after incision and drainage with fistulotomy for ischiorectal abscess. Am Surg 1997;63:686-9.

6. Amato A, Bottini C, De Nardi P, et al. Evaluation and management of perianal abscess and anal fistula: SICCR position statement. Tech Coloproctol 2020;24:127-43.

7. Adamo K, Sandblom G, Brannstrom F, et al. Prevalence and recurrence rate of perianal abscess--a populationbased study, Sweden 1997-2009. Int J Colorectal Dis 2016;31:669-73.

8. Smith SR, Newton K, Smith JA, et al. Internal dressings for healing perianal abscess cavities. Cochrane Database Syst Rev 2016:CD011193.

9. Whiteford MH. Perianal abscess/fistula disease. Clin Colon Rectal Surg 2007;20:102-9.

10. Zhao XY, Liu MY, Han XJ, et al. Clinical application evaluation study of "Guidelines for Diagnosis and Treatment of Common Diseases in Anorectal Department of Traditional Chinese Medicine". Chinese Journal of Chinese Materia Medica 2017;42:3252-6.

11. Li JY. Observation on the clinical efficacy of modified stage I IS drainage in the treatment of high perianal abscess. Modern Diagnosis and Treatment 2018;29:1184-6.

12. Dong Q, Shi SH. Research on the clinical application of thread-drawing drainage in the treatment of high anorectal abscesses. Inner Mongolia Traditional Chinese Medicine 2016;35:171-2.

13. Xu Z. Study on the therapeutic value of thread-drawing drainage method in high anorectal abscess. Electronic Journal of Cardiovascular Diseases of Integrated

Cite this article as: Shi Y, Zhi C, Cheng Y, Zheng L. A systematic review and meta-analysis of incision and seton drainage in the treatment of high perianal abscess. Ann Palliat Med 2021;10(9):9830-9840. doi: 10.21037/apm-21-2229
Traditional Chinese and Western Medicine 2020;8:186-8.

14. Ma CL, Tao X, Tang ZH. The application value of threaddrawing drainage in the treatment of high anorectal abscess. Modern Journal of Integrated Traditional Chinese and Western Medicine 2016;25:1452-4.

15. Xu Q. Analysis of the clinical effect of abscess IS method in the treatment of perianal abscess. Chinese Journal of Anorectal Diseases 2020;40:80.

16. Lin XQ, Gao XM. Clinical observation of IS contra-portal floating thread drainage in the treatment of high perianal abscess. Chinese Journal of Anorectal Diseases 2011;31:35-6.

17. Zhang RB. Clinical analysis of IS operation and ID operation in the treatment of high horseshoe perianal abscess. Shanxi Medical Journal 2017;46:918-20.

18. Yang CJ, Song XH. Observation of the curative effect of IS drainage in the treatment of high perianal abscess. Modern Medicine and Health 2017;33:3455-6.

19. Tao X. Observation on the curative effect of ID threaddrawing operation in the treatment of 32 cases of high perianal abscess. Contemporary Medicine 2013;31:64-5.

20. Wei ZH, Wei K, Zhao Y. The effect of surgical ID on the formation of anal fistula in patients with perianal abscess. Electronic Journal of Cardiovascular Diseases of Integrated Traditional Chinese and Western Medicine 2020;8:182.

21. Liu LB, Wang P. One-stage radical treatment of high perianal abscess. Journal of Liaoning University of Traditional Chinese Medicine 2007;9:100-1.

22. Li YL. Analysis of the clinical efficacy of ID operation on the left and right sides of posterior IS for high horseshoe perianal abscess. Oriental Medicinal Food 2019;17:38.

23. Song YZ. One-stage operation to radically cure fistula perianal abscess: An observation of 60 cases. Clinical Journal of Traditional Chinese Medicine 2005; 17:385-6.

24. Sun J. The effect of TCM thread-drawing therapy on high perianal abscess and its influence on anal function. Chinese and Foreign Medical Research 2019;17:32-4.

(English Language Editor: B. Meiser) 